Nine days after admission, her general health having improved, Mr. Stanley divided the tendo-Achillis by a subcutaneous incision. The limb remained upon a pillow for six days, when Stromeyer's apparatus was applied. Eleven days after this, as the foot had by the apparatus been brought to a right angle with the leg, a Scarpa's boot was applied, and the patient was allowed to get up. She was then enabled to walk by the support of a stick, which she soon laid aside.

On the 1st of August, about two months after the performance of tenotomy, a boot, with iron rods extending to just below the knee, but with a stop-joint, to prevent any contraction of the tendon, was applied; and two days afterwards she was discharged in a fair way of recovery, with directions to wear the boot for some months.

As stated above, we shall just now refrain from any remarks, merely observing that in cases of this kind it is always advantageous, be the disease feigned or real, to have recourse to any operation which cannot possibly do the patient any harm. Among operations of this kind we would almost include the application of blistering-plaster, for we saw the other day a woman at St. Thomas's Hospital, (under the care of Mr. Mackmurdo,) affected with supposed involuntary closure of the mouth, with whom repeated blistering under the chin, relaxed either the muscles or her obstinacy.

\section{SALINE INJECTIONS IN CHOLERA.}

Br G. OWEN REES, M.D., F.R.S., F.R.C.P., ASSISTANT-PHYSICIAY TO GUY'S HOSPITAL. ETC.

As the cholera has again visited this country, I venture to direct the attention of the profession to a point of considerable importance as respects the application of one of the remedies for the disease. With the view of recovering some of the worst cases, and when other remedial means have been unsuccessfully tried, practitioners have occasionally injected saline fluids into the veins. As this proceeding has been attended with great advantage in several recorded cases, and has produced in nearly all a temporary good effect, it has appeared to me that had the injected fluids been composed in more strict accordance with the chemical and physical constitution of the blood than was the case, we might have obtained advantages from the injections very far exceeding those we have on record.

With regard to the chemical constitution of the fluid, it would appear that we can scarcely venture to interfere with the organic constituents of the blood, nor imitate the animal extractives and protein compounds of the circulating fluid, in order to supply them if deficient. There is, however, no occasion for this in Asiatic cholera, for the evacuations from the intestinal surface, which destroy the healthy characters of the blood in that disease, appear to contain but little organic matter, being chiefly made up of water holding the salts of the blood in solution. Thus Vogel and Wittstock agree in describing cholera motions as containing intestinal mucus, traces of albumen, and the ordinary salts of the blood, with carbonate of soda somewhat in excess. The analysis of cholera blood again points clearly to the necessity of supplying more especially salts and water, if we desire to restore it to the heaithy standard.

With these facts before us, it is obvious that if we inject the veins at all, a solution of the salts of the blood is indicated in cases of Asiatic cholera : and it would seem well that those who may think it advisable to have occasional recourse to that plan of treatment for the recovery of severe and advanced cases, should keep a mixture of saline ingredients, combined in the proportions (or as nearly so as possible) observed in healthy blood. In order to answer the above requirements, I would recommend the following powder to be prepared, and kept ready for solution :-Chloride of sodium, three ounces; phosphate of soda, one ounce; carbonate of soda, one ounce and a half; sulphate of soda, half an ounce.

As regards the physical peculiarities of the fluid for injection, it is quite as important that its characters in this respect should be attended to as that its chemical constitution should bear its proper relations to the blood. The specific gravity and temperature of the injection will require to be carefully adjusted before we can expect to put our patient under the conditions most favourable for recovery when mixing it with the blood. With regard to temperature, but little need be said; $98^{\circ}$ Fahr. is necessary, as being that of healthy blood; and I would only remarix, that it should be determined by a correct thermometer, and not by one of those cheap instru- ments with which the market is just now unfortunately inundated. The specific gravity should be adjusted by gradually diluting a strong solution of the saline mixture above described with small portions of distilled water, at the temperature of from $58^{\circ}$ to $63^{\circ}$ Fahr, occasionally testing it with a saccharometer, (the ordinary instrument used in the examination of urine,) until the instrument indicates a specific gravity of 1030 . This being attained, the fluid has merely to be brought to the temperature of $98^{\circ} \mathrm{Fahr}$. to fit it for use.

It is not my intention to enter, on this occasion, upon the reasons why it is so absolutely necessary to attend to the specific gravity of the injected fluid, further than to state that I have adopted that of the serum of the healthy blood, which has a specific gravity such as acts on the blood corpuscles by producing a gradual endosmosis, and thus avoids the evil effects which solutions varying much from the same weight inflict on those bodies.

In conclusion, I would beg to refer those who may be interested in this question of the effects produced on the blood by solutions of varying specific gravity to a paper by Mr. Samuel Lane and myself contained in the thirteenth number of the Guy's Hospital Reports, and also to remarks having reference to the physical structure of the blood, contained in the second edition of my work on the Blood and Urine.

10, Cork-street, Burlington-gardens, Sept. 1853.

P.S.- Since writing the above I have seen, in the tenth volume of the Medical Gazette, the prescription for preparing the fluid used by Dr. Lewin and others for injecting the veins, and have ascertained, by experiment, that the specific gravity is so low as to endanger the integrity of the blood corpuscles if mixed with the blood. This danger is increased in Asiatic cholera, inasmuch as the corpuscles, owing to the drain of fluid which has occurred, contain a more concentrated fluid than in health, and will therefore be more easily destroyed by the presence of fluids of low specific gravity.

\section{PRACTICAL REMARKS ON DISEASES OF: THE EYE.}

BY JAMES DIXON, EsQ., F.R.C.S.

SUROEON TO THE ROTAL LONDON OPHTHALMIC HOSTITAL.

\section{VII.-On the Treatment of Posterior Displacement of:} THE LENS.

Displacemant of the lens backwards into the posterior chamber of the eye is an accident which is almost certain ultimately to destroy vision, by inducing slow disease of the retina. The lens also acts as a foreign body on other structures, and frequently gives rise to distressing attacks of pain, which continue year after year to harass the patient. "Why," it may be asked, "if such be the case, do we find the operation of depression recommended by surgical anthorities as a mode of treating cataract?--an operation which consists in effecting this very displacement of the lens." Fully to answer this question would lead me away from the object of the pre sent paper. I may, however, observe that the operation of depression, if actually performed according to the rules laid down by some of its advocates, is really liable to produce the same disastrous results that follow accidental displacement. The lens, which, when deprived of its capsule, so readily under goes solution and absorption, resists those changes so long as. the capsule remains entire. And, in fact, if it were possiblefor the surgeon to follow the directions for performing depression laid down by some of the older writers, and pass a needle between the iris and anterior face of the capsule without wounding either, and then thrust down the lens, in its unbroken capsule, below the level of the pupil, the lens, acting as a foreign body, would set up such morbid changes as must eventually destroy the function of the retina. Those cases of depression which prove permanently successful, turn out well in spite of the operator, who does in practice what his theory forbids, and bears the capsule, while endeavouring to displace the lens.*

It is possible for the lens to become loosened from its connexions, and sink down into the posterior chamber, without any violence having been inflicted on the eye, but merely in consequence of softening and giving way of the suspensory ligament, and a want of due consistency in the vitreous humour.t It is probable, indeed, that the first of these

* The objections here urged against depression are not altogether applicable to reclination, in which operation the capsule is supposed to be divided, both before and behind, previously to the body of the lens being displaced.

$\dagger$ For the particulars of such a case, see THE LaNcri, Feb. 1852. 